\title{
Ultrasound: novel techniques
}

\author{
Stephanie R. Wilson \\ Division of Gastroenterology, University of Calgary, Calgary, Canada
}

Ultrasound is a safe, readily available, and relatively inexpensive imaging modality. It has achieved large success for routine imaging in many arenas including obstetrics, solid abdominal organs, small parts, vascular, and emergency medicine where its excellent resolution and real-time imaging capability have made it the modality of choice for many medical problems. However, with regards to imaging requiring more sophisticated technology, US is often left in the shadow of more expensive modalities, including both CT and MR scan, where contrast enhancement, for example, has become their standard of performance.

Today, however, we witness the implementation of recently introduced revolutionary novel techniques for ultrasound which provide highly competitive performance in two major clinical arenas. The first is contrastenhanced ultrasound (CEUS), performed following the injection of a microbubble contrast agent, and allowing for the first time, the demonstration of blood flow to the perfusion level with ultrasound. The worldwide approval indication for CEUS is liver mass characterization, now standard in many countries. However, widespread offlabel use is highly successful for renal mass characterization, in the follow-up of traumatic organ injury, and for an ever increasing number of applications. Pediatric CEUS will reduce the demand for ionizing radiation in children. The second novel technique involves US elastography, providing noninvasive measurement of tissue stiffness with its biggest contribution to the determination of liver fibrosis in those with suspect chronic liver disease. Further, a single manuscript addresses the field of machine learning for ultrasound, a form of artificial intelligence showing the potential value of data and pattern recognition for improved diagnosis.

This special issue of Abdominal Radiology, devoted to the implementation of these exiting new ultrasound technologies, is far reaching, touching especially on the growing clinical applications of CEUS and elastography. Their addition will surely escalate the role of ultrasound to previously unachievable heights. 